\title{
Structural properties of crystallizable polymer melts: Intrachain and interchain correlation functions
}

\author{
Thomas Vettorel, ${ }^{*}$ Hendrik Meyer, ${ }^{\dagger}$ and Jörg Baschnagel \\ Institut Charles Sadron, CNRS-UPR22, 6 Rue Boussingault, 67083 Strasbourg, France \\ Matthias Fuchs \\ Fachbereich Physik, Universität Konstanz, 78457 Konstanz, Germany
}

(Received 5 October 2006; published 10 April 2007)

\begin{abstract}
We present results from constant pressure molecular-dynamics simulations for a bead-spring model of a crystallizable polymer melt. Our model has two main features, a chemically realistic intrachain rigidity and a purely repulsive interaction between nonbonded monomers. By means of intrachain and interchain structure factors we explore polymer conformation and melt structure above and below the temperature $T_{\text {crys }}^{\text {hom }}$ of homogeneous crystallization. Here, we do not only determine average spatial correlations, but also site-specific correlations which depend on the position of the monomers along the polymer backbone. In the liquid phase above $T_{\text {crys }}^{\text {hom }}$ we find that this site dependence can be well-accounted for by known theoretical approximations, the Koyama distribution for the intrachain structure and the polymer reference interaction site model (PRISM) for the interchain structure. This is no longer true in the semicrystalline phase. Below $T_{\text {crys }}^{\text {hom }}$ short chains fully extend upon crystallization, whereas sufficiently long chains form chain-folded lamellae which coexist with amorphous regions. The structural features of these polymer crystals lead to violations of premises of the Koyama approximation or PRISM theory so that both theoretical approaches cannot be applied simultaneously. Furthermore, we find a violation of the Hansen-Verlet freezing criterion; our polymer melt crystallizes more easily than a simple liquid. This hints at the importance of the coupling between conformation (backbone rigidity) and density (packing constraints) for polymer crystallization.
\end{abstract}

DOI: 10.1103/PhysRevE.75.041801

PACS number(s): 61.25.Hq, 61.20.Ja

\section{INTRODUCTION}

When supercooling a crystallizable polymer melt the melt undergoes a transition to a semicrystalline state [1]. This polymer crystal is characterized by a hierarchy of ordered structures. The basic units are chain-folded lamellae, consisting of regularly packed, extended chain sections. The lamellae interleave with amorphous regions to form layers, which in turn organize themselves in larger scale superstructures (spherulites).

It is commonly believed that this hierarchical structure is the result of kinetic effects which predominate crystallization of the supercooled polymer melt $[2,3]$. This point of view is supported by various observations. For instance, the lamellar thickness $(\approx 10 \mathrm{~nm})$ is found to be much smaller than values predicted by equilibrium considerations $[1,4,5]$; the melting behavior of the crystal depends on thermal history [6]; and true thermodynamic coexistence of the polymer liquid and crystalline phase does not seem to exist because the crystallization temperature is generally observed to be lower than the melting temperature [1].

It is thus not surprising that great effort has been invested in exploring the features of the metastable polymer melt (see, e.g., Refs. $[2,7]$ for reviews). Important problems that have been addressed include the crystallization mechanism in the very early stage (nucleation-and-growth [4], spinodal-

\footnotetext{
*Present address: Max-Planck Institut für Polymerforschung, Ackermannweg 10, 55128 Mainz, Germany.

†Corresponding author. Email address: hmeyer@ics.u-strasbg.fr
}

assisted crystallization [8]), the criteria for spontaneous selection of the lamellar thickness [4,5], or the molecular processes responsible for the growth of the crystal front [3].

In addition to these studies of the metastable melt, attempts have also been made to tackle the problem of polymer crystallization "from the liquid" - that is, to understand the thermodynamic driving force leading to the liquid-solid transition on cooling. For instance, density-functional theories (DFTs) were developed [9,10]. Quite generally, DFT [11] is a method to predict equilibrium features of an inhomogeneous system (the crystal) from information about the structure of the homogeneous system (the liquid), provided an accurate free-energy functional is known for the considered system and the required structural input is available with sufficient precision. DFT approaches to polymer crystallization $[9,10]$ utilized an approximate description of the conformation and structure of the polymer liquid. A critical test of the proposed free-energy functional could thus be achieved only partially. Here, we demonstrate that this approximate description of the liquid structure is not (or no longer) necessary. We carried out molecular-dynamics simulations of a coarse-grained model for a crystallizable polymer melt and show that the pertinent intramolecular and intermolecular correlation functions can be determined over a large range of wave vectors with high statistics. Our work could thus inspire further developments of DFT, eventually leading to an accurate equilibrium free energy functional for polymer liquids. Such an accurate functional is a prerequisite for an extension of DFT to dynamical problems, such as the growth of new phases or other out-of-equibirium processes $[11,12]$.

The outline of this paper is as follows. First we discuss the simulation model and present some details about the 

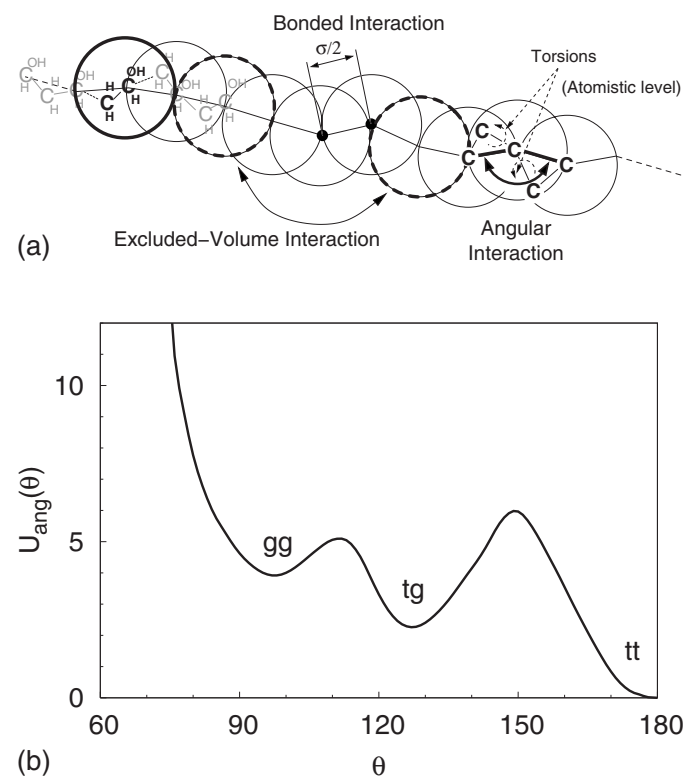

FIG. 1. Schematic representation of the correspondence between the CG-PVA model and the underlying atomistic PVA chain. At the coarse-grained level, the interaction potentials for chain connectivity, excluded volume, and chain stiffness are indicated. The angular potential $U_{\text {ang }}(\theta)$ (in units of $k_{\mathrm{B}} T$ ) is shown as a function of the bond angle $\theta$ (in degrees). The minima of $U_{\text {ang }}(\theta)$ reflect specific states of two successive torsions at the atomistic level $(\mathrm{tt}=$ trans-trans, $\mathrm{tg}=$ trans-gauche, and $\mathrm{gg}=$ gauche - gauche $)$.

simulation technique (Sec. II). This is followed by a brief introduction to the quantities used to explore the structure of our model above and below the temperature of homogeneous crystallization. Section IV then discusses the corresponding results obtained for-averaged and monomer-resolvedintrachain and interchain correlation functions. Section V summarizes the main findings of our work.

\section{MODEL AND SIMULATION}

Our studies utilize a bead-spring model obtained by a controlled coarse-graining procedure [13] from atomistic simulations of poly(vinyl alcohol) (PVA). In this section, we briefly describe this coarse-grained (CG) model, referred to as "CG-PVA model" in the following, and discuss its strengths and limitations. More details may be found in Refs. [14-17].

\section{A. The CG-PVA model}

Figure 1 illustrates the link between the CG-PVA and PVA models. A monomer of the coarse-grained chain corresponds to a monomer of PVA. This identification forces consecutive monomers along the CG-PVA chain to overlap. The average bond length $b_{0}$ must thus be smaller than the monomer diameter of PVA. Fluctuations of the bond length $b$ about $b_{0}=\sigma / 2=0.26 \mathrm{~nm}-\sigma=0.52 \mathrm{~nm}$ roughly agrees with the monomer diameter of PVA - are restricted by a harmonic potential,

$$
U_{\text {bond }}(b)=\frac{1}{2} k_{\text {bond }}\left(b-b_{0}\right)^{2} \text {. }
$$

The fluctuations of $b$ are small because the force constant $k_{\text {bond }}$ is large, $k_{\text {bond }}=2704 k_{\mathrm{B}} T / \sigma^{2}$.

Monomers of distinct chains and of the same chain if they are three bonds or more apart interact by a 9-6 LennardJones (LJ) potential,

$$
U_{\mathrm{LJ}}(r)= \begin{cases}\varepsilon_{0}\left[\left(\frac{\sigma_{0}}{r}\right)^{9}-\left(\frac{\sigma_{0}}{r}\right)^{6}\right]+C, & r \leq r_{\mathrm{cut}}, \\ 0, & r>r_{\mathrm{cut}} .\end{cases}
$$

Here, $\sigma_{0}=0.89 \sigma=0.46 \mathrm{~nm}, \varepsilon_{0}=1.511 k_{\mathrm{B}} T$, and $C=4 \varepsilon_{0} / 27$ is chosen such that $U_{\mathrm{LJ}}\left(r_{\text {cut }}\right)=0$ for the cutoff distance $r_{\text {cut }}$ $=(3 / 2)^{1 / 3} \sigma_{0}(\simeq 1.02 \sigma), r_{\text {cut }}$ being the minimum of the LJ potential. With the latter choice, nonbonded interactions are purely repulsive.

In addition to bond and excluded-volume interactions, a potential $U_{\text {ang }}(\theta)$ is also associated with the angle $\theta$ between consecutive $\mathrm{CG}$ bonds. $U_{\text {ang }}(\theta)$ is determined directly from atomistic simulations by Boltzmann-inversion of the probability distribution $P_{\text {ang }}(\theta)$, that is, by posing $P_{\text {ang }}(\theta)$ $\propto \sin \theta \exp \left[-U_{\text {ang }}(\theta) / k_{\mathrm{B}} T\right]$. Figure 1 depicts the resulting bond angle potential. $U_{\text {ang }}(\theta)$ clearly reveals the fingerprint of the underlying PVA chain. Since the bond angle of CGPVA is determined by two successive torsions at the atomistic level (1), $U_{\text {ang }}(\theta)$ has three minima corresponding to the three energetically favorable states, trans-trans, transgauche, and gauche-gauche. $U_{\text {ang }}(\theta)$ thus confers a semiflexible character to the CG-PVA chains with a flexibility mechanism such as in the rotational-isomeric-state (RIS) model $[18,19]$. This feature becomes important for the structure of the melt in the semicrystalline state.

\section{B. Discussion of the model}

\section{Units}

The coarse-graining procedure described in Ref. [13] and further discussed in Ref. [17] allows one to adjust the parameters of the CG potentials so as to reproduce (some) conformational and structural features of the underlying atomistic model. The optimization is carried out at a specific thermodynamic state point, characterized, e.g., by some temperature $T$ and pressure $p$. For PVA, $T=550 \mathrm{~K}$ and $p=1$ bar were chosen at the atomistic level. This corresponds to $T=1$ and $p=8$ for CG-PVA. Here, we employed reduced units, that is, energies are measured in units of $k_{\mathrm{B}} T$ (with the Boltzmann constant $\left.k_{\mathrm{B}}=1\right)$, lengths in units of $\sigma(=1)$, and time in units of $\tau=\sigma \sqrt{m / k_{\mathrm{B}} T}$ (with the monomer mass $m=1$ ). These reduced units will be used in the following.

\section{Strengths and limitations of the model}

The CG-PVA model has advantages and drawbacks. A drawback certainly is that chemical specificity is lost. The identification "1 monomer of PVA $\Leftrightarrow 1$ bead of CG-PVA" does not allow one to describe features which are directly related to monomer properties of PVA. This implies, for in- 


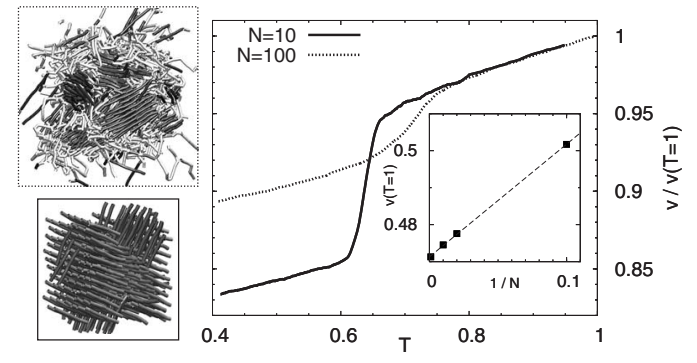

FIG. 2. Main figure: Phase diagram of CG-PVA, i.e., specific volume $v$ versus temperature $T$, for two chain lengths, $N=10$ (full line) and 100 (dotted line). (The volume is normalized by its value at $T=1$.) Crystallization manifests itself by a drop of $v(T)$. The phase transition is sharper for $N=10$ than for $N=100$. For short chains $N=10$, crystallization leads to the formation of a crystal of fully stretched chains (cf. lower snapshot). For $N=100$, on the other hand, a lamellarlike structure is obtained (cf. upper snapshotcrystalline domains are emphasized by the darker lines). Inset: Dependence of $v(T)$ on chain length for $T=1$ (fluid state). We find that the volume varies linearly with $1 / N$; this chain length dependence may be associated with the chain ends (see, e.g., [45]).

stance, that the crystal structure of PVA cannot be reproduced. Stretched parts of the CG-PVA chains have cylindrical symmetry which leads to hexagonal ordering (see Sec. IV C 3), instead of to a monoclinic crystalline phase characteristic of PVA [20]. In some cases, a further drawback might be that attractive or electrostatic interactions, present at the atomistic level, are not explicitly incorporated in the model; they only enter implicitly into the potential parameters through the mapping procedure. So, situations where these interactions are important cannot be explored (for instance, attractive interactions must be included for the simulation of polymer-air interfaces).

On the other hand, we feel that, for the study of polymer crystallization, the CG-PVA model has two main strengths, one computational, the other related to the physics of polymer crystallization. Computationally, the model is convenient. The simple monomer structure and the short-range potentials allow for an efficient simulation of large systems. The study of large systems is important to minimize finitesize effects on the structure formation upon crystallization (for further discussion see Ref. [15]). From a physical point of view, the model provides the interesting insight that we can suppress monomer-monomer attractions and still observe key features of polymer crystallization [14-16]. The competition of packing constraints and the RIS-like chain stiffness, both of which increase on cooling, is sufficient to reproduce the following, experimentally well-known facts $[4,7]$. (i) Short chains fully extend when crystallizing, while long chains fold back on themselves, forming lamellarlike structures (cf. Fig. 2). Detailed analysis of the chain-folded lamellae reveals the fingerprint of the angular potential $U_{\text {ang }}(\theta)$ [15]. The folds consist of trans-gauche and gauche-gauche states. Apparently, the secondary minima of $U_{\text {ang }}(\theta)$ kinetically stabilize these states and thus assist chain folding. (ii) The thickness $d$ of the lamellae is inversely proportional to the crystallization temperature $T_{\text {crys }}$ of isothermal relaxation experiments (i.e., an amorphous melt is quenched to $T_{\text {crys }}$ below the melting point and then relaxed isothermally). Lamellae of a given thickness melt at a specific temperature $T_{\mathrm{m}}(d)>T_{\text {crys }}(d) . T_{\mathrm{m}}(d)$ is also inversely proportional to $d$. (iii) For continuous cooling and heating cycles, the homogeneous crystallization and melting temperatures increase with chain length $N$. The qualitative features of this increase agree well with experimental results on short alkanes $[16,17]$.

\section{Simulation aspects}

We analyze polymer melts of $n$ linear chains consisting of $N$ monomers each. More precisely, the studied values of $N$ and $n$ are $N=10(n=288), N=50(n=72)$, and $N=100(n$ $=192$ ). Our results are obtained from constant pressure simulations ( $p=8$; Berendsen barostat [21]) at various temperatures $T$ (Langevin thermostat [21]).

We explore structural properties of the liquid phase for $T \geq 0.8$ and of the crystalline phase at $T=0.4$. The lower bound, $T=0.8$, of the liquid phase is determined by the temperature $T_{\text {crys }}^{\text {hom }}$ where homogeneous nucleation occurs. For $N=100$, Refs. $[15,16]$ found $T_{\text {crys }}^{\mathrm{hom}}=0.78$. As we expect $T_{\text {crys }}^{\text {hom }} \leq 0.78$ for $N<100$, the choice $T \geq 0.8$ for the liquid phase should be appropriate for all $N$ studied. We thus refer to $T_{\text {crys }}^{\text {hom }}=0.78$ as "onset temperature of homogeneous crystallization" in the following.

In the liquid phase, we analyze time series of fully equilibrated configurations at each $T$. The configurations of the crystalline phase are obtained by continuous cooling from $T=1$ to 0.4 with the rate $5 \times 10^{-6}$, followed by isothermal relaxation at temperature $T$. A systematic variation of the cooling rate $\Gamma$, from $2 \times 10^{-5}$ to $5 \times 10^{-7}$, was carried out for $N=10$. As expected, with decreasing $\Gamma$ the transition from the liquid to the crystal becomes sharper and the structure of the crystalline phase better ordered. For $N=100$, however, the impact of the cooling rate appears to be weak (at least for the rates studied here). Certainly, a crystal of fully stretched chains, as for $N=10$ (see Fig. 2), is not easily accessible in that case, even for slow cooling, and $v(T)$ always exhibits a rather smooth transition to the (semi-) crystalline phase.

\section{ANALYZED QUANTITIES AND TECHNICAL DETAILS}

\section{A. Static structure factors}

One way to characterize the structure of a melt is to explore spatial correlations between two monomers $a$ and $b$ via density-density correlation functions. In this approach, the basic correlation function is the monomer-monomer (or sitesite) structure factor $S_{a b}(\mathbf{q})$ which is defined by

$$
S_{a b}(\mathbf{q})=\frac{1}{n} \sum_{i, j=1}^{n}\left\langle\exp \left[-i \mathbf{q} \cdot\left(\mathbf{r}_{i}^{a}-\mathbf{r}_{j}^{b}\right)\right]\right\rangle .
$$

Here, $\mathbf{q}$ is the wave vector in reciprocal space, $\mathbf{r}_{i}^{a}$ denotes the position of monomer $a(=1, \ldots, N)$ in chain $i(=1, \ldots, n)$, and the angular brackets represent the average over all configurations of the melt.

For spatially homogeneous and isotropic systems, such as a polymer melt in the liquid phase for $T>T_{\text {crys }}^{\mathrm{hom}}, S_{a b}(\mathbf{q})$ only 
depends on the modulus $q$ of the wave vector. In order to compare liquid and crystalline phases we will also, for $T<T_{\text {crys }}^{\text {hom }}$, only determine structure factors that are spherically averaged over all wave vectors with the same modulus $q$ (as in powder diffraction experiments).

$S_{a b}(q)$ can be split into intrachain and interchain contributions

$$
S_{a b}(q)=w_{a b}(q)+\rho_{\mathrm{ch}} h_{a b}(q),
$$

where

$$
\rho_{\mathrm{ch}}=\frac{n}{V}
$$

is the chain density $[V=$ volume of the (periodic) simulation box]. The intrachain contribution is given by

$$
w_{a b}(q)=\frac{1}{n} \sum_{i=1}^{n}\left\langle\exp \left[-i \mathbf{q} \cdot\left(\mathbf{r}_{i}^{a}-\mathbf{r}_{i}^{b}\right)\right]\right\rangle
$$

and the interchain contribution by

$$
\rho_{\mathrm{ch}} h_{a b}(q)=\frac{1}{n} \sum_{i \neq j}^{n}\left\langle\exp \left[-i \mathbf{q} \cdot\left(\mathbf{r}_{i}^{a}-\mathbf{r}_{j}^{b}\right)\right]\right\rangle .
$$

The static structure factor measured in scattering experiments is recovered by averaging over all monomer pairs $(a, b)$

$$
S(q)=\frac{1}{N} \sum_{a, b=1}^{N} S_{a b}(q)=w(q)+\rho_{\mathrm{m}} h(q),
$$

where $\rho_{\mathrm{m}}=N \rho_{\mathrm{ch}}$ is the monomer density and

$$
w(q)=\frac{1}{N} \sum_{a, b=1}^{N} w_{a b}(q), \quad h(q)=\frac{1}{N^{2}} \sum_{a, b=1}^{N} h_{a b}(q) .
$$

Here, $w(q)$ is the intrachain structure factor (form factor [22]) of a chain and $h(q)$ is the Fourier transform of the site-averaged intermolecular pair-correlation function [23].

Commonly only the average structure factors $S(q)$ and $w(q)$ are considered. In the following, we also discuss siteresolved quantities, such as $S_{a b}(q)$ and $w_{a b}(q)$. One aim of our study is to understand to what extent specific monomer correlations deviate from the average behavior.

\section{B. Technical details}

The computation of structure factors from simulation requires a large number of (independent) configurations in order to minimize statistical errors. This is particularly important for site-resolved quantities [24] where averages along the chain cannot be carried out due to the explicit dependence on the monomer indices $(a, b)$. For $T>T_{\text {crys }}^{\text {hom }}$ we averaged the structure factors over 360 configurations for $N=10$ (the system consists of 288 chains), 400 configurations for $N=50$ (72 chains), and 1000 configurations for $N$ $=100$ (192 chains). For $T<T_{\text {crys }}^{\text {hom }}$ about 300 configurations were used for statistical averaging.

The structure factors were calculated as functions of modulus $q$ of the wave vector $\mathbf{q}$. Only wave vectors compat-

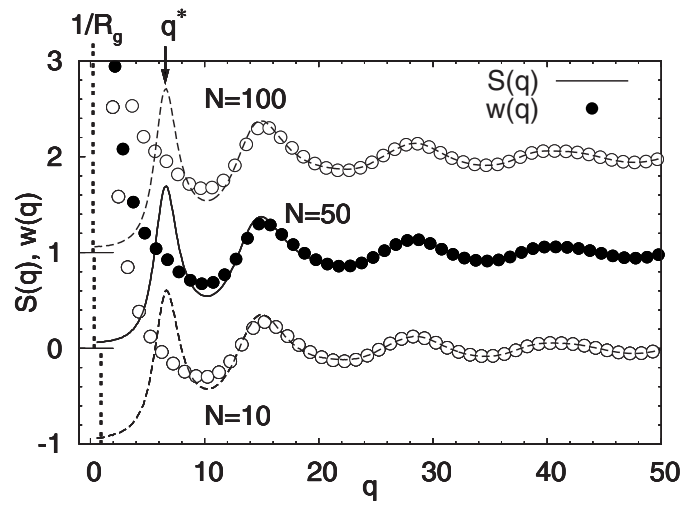

FIG. 3. Structure factor $S(q)$ (lines) and form factor $w(q)$ (circles) versus $q$ at $T=1$ for $N=10,50$, and 100. The data for $N=10$ and 100 are shifted for clarity (by -1 for $N=10$, by +1 for $N=100$ ). The vertical arrow indicates the value of $q$ associated with the amorphous halo $q^{*}$ and the vertical dashed lines the radii of gyration, $R_{\mathrm{g}} \simeq 1.08$ for $N=10, R_{\mathrm{g}} \simeq 3.11$ for $N=50$, and $R_{\mathrm{g}} \simeq 4.46$ for $N=100$. The position and the amplitude of the amorphous halo slightly depend on $N: q^{*} \simeq 6.65, S\left(q^{*}\right) \simeq 1.61$ for $N=10 ; q^{*} \simeq 6.55$, $S\left(q^{*}\right) \simeq 1.71$ for $N=100$.

ible with the periodic boundary conditions of the cubic simulation box were used in this calculation. For boxes of linear dimension $L$ this implies the following lower bounds on the $q$ value: $q_{\min }=0.56 \quad(L=11.31$ for $N=10), \quad q_{\min }=0.53$ $(L=11.98$ for $N=50)$, and $q_{\min }=0.30(L=20.89$ for $N=100)$.

\section{STATIC PROPERTIES OF THE LIQUID AND CRYSTALLINE STATES}

\section{A. Average structure factors at high temperature: An overview}

Figure 3 compares the structure factor $S(q)$ with the form factor $w(q)$ for three chain lengths, $N=10,50$, and 100, at $T=1$. At this temperature, $S(q)$ displays features characteristic of the liquid state. For all $N$ the structure factor is small at low $q$, reflecting the weak compressibility $\kappa_{T}$ of the melts. With increasing $q, S(q)$ increases toward the "amorphous halo," the first maximum occurring at $q^{*}$, and then exhibits oscillations whose amplitude decreases and eventually converges to 1 as $q \rightarrow \infty$. Figure 3 shows that this large- $q$ behavior is entirely intramolecular. For $q \geq 20, S(q)$ agrees with the form factor $w(q)$ and so the intermolecular correlation function $h(q)$ vanishes (see Fig. 4). On the other hand, intermolecular correlations strongly contribute for smaller $q$, in particular for $q \lesssim q^{*}$. The different importance of intrachain and interchain contributions above and below $q^{*}$ is a property of the CG-PVA polymer liquid, which bead-spring models without $U_{\text {ang }}(\theta)$ do not necessarily exhibit (for $q>q^{*}$ both intrachain and interchain contributions are important [25]). CGPVA shares this property with atomistic models of polymer melts (see, e.g., $[26,27]$ ).

Further insight into the interchain structure of the CGPVA model can be obtained by comparing $h(q)$ with an approximation motivated by the theory of simple liquids. Since the particles of a simple liquid have no internal structure, we have $w(q)=1$ and so $\rho_{\mathrm{m}} h(q)=S(q)-1$ [23] 

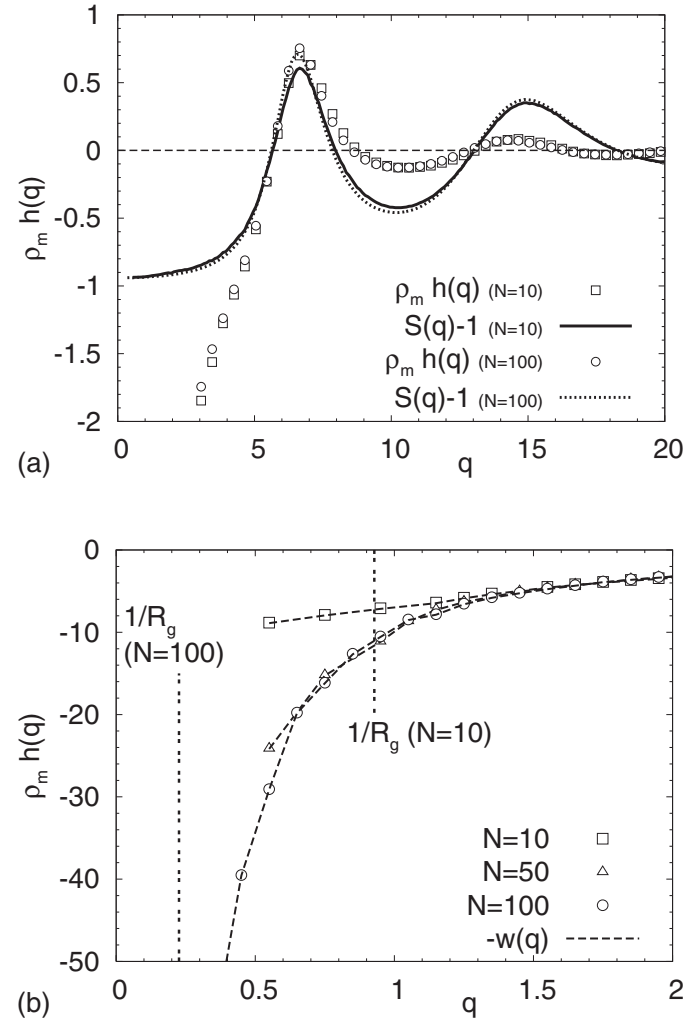

FIG. 4. Interchain structure factor $h(q)$ versus $q$ for $T=1$ and various $N$. The upper panel compares $\rho_{\mathrm{m}} h(q)$ (symbols) for $N=10$ and 100 to the "simple-liquid approximation" $S(q)-1$ (lines). The lower panel magnifies the behavior of $\rho_{\mathrm{m}} h(q)$ (symbols) for small $q$. Here, three chain lengths are shown, $N=10,50$, and 100. For all $N$ the dashed lines represent the negative of the corresponding intrachain structure factor, $-w(q)$ (cf. Fig. 3). The vertical dotted lines indicate the $q$-values corresponding to $1 / R_{\mathrm{g}}\left(R_{\mathrm{g}} \simeq 1.08\right.$ for $N=10$ and $R_{\mathrm{g}} \simeq 4.46$ for $N=100$ ).

Figure 4 compares $\rho_{\mathrm{m}} h(q)$ to $S(q)-1$ to stress similarities and differences of our polymer melt compared to a simple liquid. We find that $\rho_{\mathrm{m}} h(q)$ and $S(q)-1$ closely agree with one another around the amorphous halo, i.e., for $q=q^{*}$ $\approx 6.6$. This finding is not unreasonable. The first peak of $S(q)$ characterizes the packing of monomers in the melt. It is dominated by interchain contribution and should thus be similar to that of a simple liquid of monomers. Interestingly, the agreement between $\rho_{\mathrm{m}} h(q)$ and $S(q)-1$ appears to improve as the chain length increases.

For $q \gg q^{*}$, however, $S(q)$ is fully determined by intrachain effects, as pointed out before in the discussion of Fig. 3. Thus the simple-liquid approach, $\rho_{\mathrm{m}} h(q)=S(q)-1$, must deviate from the simulation data as $q$ increases beyond $q^{*}$. The approach must also break down in the opposite limit of small $q$ due to a polymer-specific effect, the "correlation hole" [28]. The term "correlation hole" means that the probability of finding a monomer of another chain in the typical volume occupied by a particular chain is decreased. Thus the correlation between monomers of different chains drops with increasing distance. This drop largely compensates the increase of $w(q)$ for small $q$ so that the sum of both intra- and interchain contributions yields a small finite value for $S(q)$ as

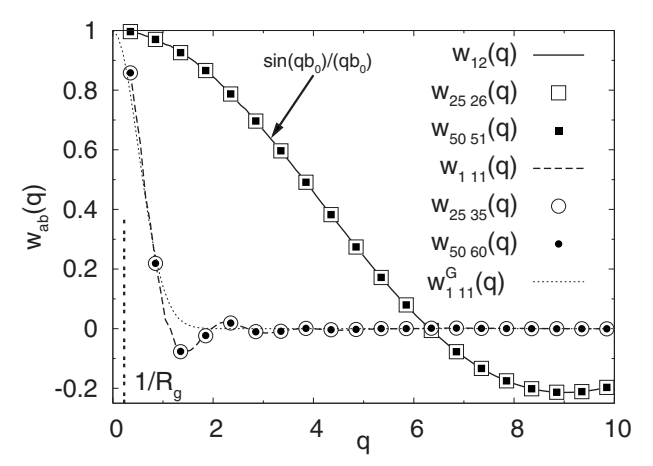

FIG. 5. Site-resolved intrachain structure factor $w_{a b}(q)$ versus $q$ for $N=100$ and $T=1$. The figure illustrates the behavior of $w_{a b}(q)$ by examples for the monomer pair $(a, b=a+n)$ with $n=1$ and 10 . For each $n$ three pairs are shown, corresponding to the end monomer $(a=1)$, the middle monomer $(a=50)$, and a monomer inbetween $(a=25)$. The dotted lines represent the Gaussian approximation, Eq. (11). The nearest-neighbor approximation, Eq. (10), is also indicated; it is indistinguishable from the simulation data for $w_{12}(q)$. The dashed vertical line marks the $q$-value associated with the radius of gyration $\left(R_{\mathrm{g}} \simeq 4.46\right)$.

$q \rightarrow 0$, corresponding to weak compressibility of the melt. Indeed, Fig. 4 reveals that $\rho_{\mathrm{m}} h(q) \approx-w(q)$.

In the next sections we will address the intrachain and interchain correlation functions in more detail, focusing in particular on their site-dependence in the liquid and crystalline phases of the melt. Such detailed information about the structure might prove important for the further development of density-functional approaches to polymer crystallization $[9,10]$.

\section{B. Intrachain structure factors}

Following Sec. III A site-resolved structure factors are the basic quantities from which averages, such as $w(q)$, may be obtained. So we begin our discussion of the intrachain structure by an analysis of the site-dependent form factor, $w_{a b}(q)$.

Figure 5 depicts $w_{a b}(q)$ at $T=1$ for $N=100$ and various combinations of the monomer pair $(a, b)$. We find that $w_{a b}(q)$ does not depend explicitly on the site index, but only on the number of bonds, $|a-b|$, between the sites $a$ and $b$. This feature is found for all chain lengths studied $(N=10,50$, and 100) and also for other bead-spring models [24,29]. It implies that the position of the monomer pair with respect to the chain end has a negligible influence on the spatial correlation between the sites $a$ and $b$.

For the further interpretation of $w_{a b}(q)$ two limits are interesting, the limit of small and large contour length $|a-b|$. Spatial correlations of adjacent monomers along the chain $(|a-b|=1)$ are determined by the bond potential of the CGPVA model [Eq. (1)]. Since this potential is rather stiff, it forces the bond length to remain close to the mean value $b_{0}$. So we expect $w_{a b=a+1}(q)$ to be given by

$$
w_{a a+1}(q)=\left\langle\frac{\sin \left(q\left|\mathbf{r}^{a}-\mathbf{r}^{a+1}\right|\right)}{q\left|\mathbf{r}^{a}-\mathbf{r}^{a+1}\right|}\right\rangle \simeq \frac{\sin \left(q b_{0}\right)}{q b_{0}} .
$$

(Here and in the remainder of this section on intrachain properties we suppress the chain index $i$ on the monomer posi- 
tions $\mathbf{r}_{i}^{a}$, see Sec. III A.) Indeed, Fig. 5 reveals that Eq. (10) is in very good agreement with the simulation data. On the other hand, for large $|a-b|$ microstructure effects, reflecting some stiffness of the chains on local scales, should no longer be dominant. We then expect that the result for ideal Gaussian chains [22],

$$
w_{a b}^{G}(q)=\exp \left(-q^{2}|a-b| \ell^{2} / 6\right), \quad \ell^{2}=\frac{6 R_{\mathrm{g}}^{2}}{N},
$$

represents a viable approximation for $w_{a b}(q)$, provided length scales much larger than $1 / q^{*}$ are considered.

Figure 5 compares $w_{a b}^{G}(q)$ to the simulated $w_{a b}(q)$. As a representative example for internal correlations with $|a-b|>1$, the figure shows data for $|a-b|=10$. We find that Eq. (11) is in reasonable agreement with the simulation only for $q \lesssim 1$. For larger $q$, deviations occur. While $w_{a b}^{G}(q)$ decays monotonically, the simulation results display oscillations, similar to the nearest-neighbor correlations $w_{a a+1}(q)$, but strongly damped in the present case. Since the oscillations of $w_{a a+1}(q)$ result from the rodlike behavior of a bond, i.e., from the "rigidity" of the chain on this length scale, we may speculate that the behavior of $w_{a b}(q)$ for large contour length, which is not captured by the Gaussian model, is related to the intrinsic stiffness of the CG-PVA chains. We pursue this idea in the following section.

\section{Koyama distribution and $w_{a b}(q)$}

The bond angle potential (Fig. 1) confers a semiflexible character to the CG-PVA model. Inspired by work on similar models $[30,31]$ we thus attempt to describe $w_{a b}(q)$ by the Koyama distribution $[27,32,33]$

$$
w_{a b}^{K}(q)=\frac{\sin B_{a b} q}{B_{a b} q} \exp \left(-A_{a b}^{2} q^{2}\right),
$$

where $\left(r_{a b}=\left|\mathbf{r}^{a}-\mathbf{r}^{b}\right|\right)$

$$
\begin{gathered}
A_{a b}^{2}=\frac{1-C_{a b}}{6}\left\langle r_{a b}^{2}\right\rangle, \quad B_{a b}^{2}=C_{a b}\left\langle r_{a b}^{2}\right\rangle, \\
C_{a b}^{2}=\frac{1}{2}\left[5-3 \frac{\left\langle r_{a b}^{4}\right\rangle}{\left\langle r_{a b}^{2}\right\rangle^{2}}\right] .
\end{gathered}
$$

Originally, the Koyama distribution was proposed as an approximation to the distribution of internal distances $r_{a b}$ for the wormlike chain model $[32,33]$. As noted by Mansfield [33], the Koyama distribution is not limited to wormlike chains, but can be applied to any semiflexible chain model for which the second and fourth moments of $r_{a b}$ are known (provided $C_{a b}^{2} \geq 0$ ).

We determined these moments and compared Eq. (12) to our simulation data. Figure 6 illustrates the results of this comparison for $N=100$ and $T=1$. Clearly, the Koyama distribution represents a substantial improvement over Eq. (11); it provides a very good description of $w_{a b}(q)$ for all $|a-b|$ in the studied $q$ range. Small deviations were only observed for $N=10$ in the case of the most remote monomers, i.e., for $|a-b| \rightarrow N-1$. This suggests that the intrachain structure of



FIG. 6. Comparison of site-resolved structure factors $w_{a b}(q)$ (lines) with the Koyama approximation, Eqs. (12) and (13) (circles). The examples shown are representative for all monomer pairs, although they always involve the end monomer $(a=1)$. The simulation data refer to $N=100$ and $T=1$. The vertical dashed line indicates the $q$-value associated with the radius of gyration $\left(R_{\mathrm{g}} \simeq 4.46\right)$.

CG-PVA in the melt can be accurately predicted by using only the first two even moments, $\left\langle r_{a b}^{2}\right\rangle$ and $\left\langle r_{a b}^{4}\right\rangle$, of the distance distribution between monomers.

Since $\left\langle r_{a b}^{4}\right\rangle$ is always larger than or equal to $\left\langle r_{a b}^{2}\right\rangle^{2}$, the key parameter $C_{a b}$ of the Koyama distribution obeys the inequality $C_{a b}^{2} \leq 1$. The equality holds if $\left\langle r_{a b}^{4}\right\rangle=\left\langle r_{a b}^{2}\right\rangle^{2}$. This is the case for rodlike behavior where $r_{a b}=|a-b| b_{0}$. Then, we recover Eq. (10) from Eqs. (12) and (13) for $b=a+1$. On the other hand, $C_{a b}$ vanishes if the internal distances $r_{a b}$ are Gaussian distributed. Therefore $C_{a b}$ measures deviations from Gaussian behavior, and we may refer to it as a nonGaussian parameter [23]. If $C_{a b}$ vanishes, Eq. (12) gives back Eq. (11) provided we write $\left\langle r_{a b}^{2}\right\rangle=|a-b| \ell^{2}$. The Koyama distribution thus interpolates between the expected small-scale [Eq. (10)] and large-scale behaviors [Eq. (11)] of our model.

The dependence of $C_{a b}^{2}$ and $\left\langle r_{a b}^{2}\right\rangle$ on the contour length $|a-b|$ is presented in Fig. 7 for $N=100$ and $T=1$. With increasing $|a-b|, C_{a b}^{2}$ continuously decreases and the meansquare internal distance tends towards $\left\langle r_{a b}^{2}\right\rangle \propto|a-b| b_{0}^{2}$. Deviations from Gaussian behavior thus diminish as larger contour lengths are considered. However, for $N=100$, they do not fully vanish, even if $|a-b|=N-1$.

In the present case, these deviations can be interpreted as a microstructure effect. By invoking the Flory ideality hypothesis $[18,28]$ we can assume that intrachain excluded volume forces are screened in the CG-PVA melt. It should thus be possible to calculate the intrachain structure from an "ideal" single chain model [18]. Here, a generalized freely rotating chain (GFRC) model appears to be appropriate because it resembles our CG-PVA model in the following respect [34]: its conformational properties are determined by the bond angle $\theta$ which fluctuates according to some distribution $P(\theta)$. For the GFRC model, the second and fourth moments depend only on $\langle\cos \theta\rangle$ and $\left\langle\cos ^{2} \theta\right\rangle$ (see Appendix B). We determined these averages from the simulation and calculated $C_{a b}^{2}$ and $\left\langle r_{a b}^{2}\right\rangle$ according to Eq. (13) and the formulas of Appendix B. The results of this calculation are included in Fig. 7 (solid lines). In fact, good agreement be- 




FIG. 7. Non-Gaussian parameter $C_{a b}^{2}$ [Eq. (13)] and meansquare internal distance $\left\langle r_{a b}^{2}\right\rangle$ versus contour length $|a-b|$. The simulation data for $N=100$ and $T=1$ are shown by circles. Since $C_{a b}^{2}$ and $\left\langle r_{a b}^{2}\right\rangle$ depend only on $|a-b|$, the figure shows results from a gliding average over all monomers $a$ separated by $|a-b|$ from monomer $b$ (i.e., $b=a+|a-b|$ ). $\left\langle r_{a b}^{2}\right\rangle$ is divided by $|a-b| b_{0}^{2}$, where $b_{0}=1 / 2$ is the average bond length, because Gaussian behavior, i.e., $\left\langle r_{a b}^{2}\right\rangle=C_{\infty}|a-b| b_{0}^{2}\left(C_{\infty}\right.$ is the characteristic ratio [18]), is expected for $|a-b| \rightarrow N$ in the large- $N$ limit. The solid lines indicate the theoretical results for $C_{a b}^{2}$ and $\left\langle r_{a b}^{2}\right\rangle$ from the generalized freely rotating chain model (cf. Appendix B).

tween the GFRC predictions and the simulation data is found [35].

\section{Intrachain properties at lower temperature}

The preceding discussion was concerned with temperatures much larger than the onset temperature of homogeneous crystallization $T_{\text {crys }}^{\text {hom }}(=0.78)$. In this section, we focus on temperatures closer to and below $T_{\text {crys }}^{\text {hom }}$.

(a) Non-Gaussian parameter $C_{a b}$. Figure 8 depicts the non-Gaussian parameter $C_{a b}^{2}$ for various $N$ and illustrates its temperature dependence. As expected, deviations from Gaussianity are stronger for shorter chains-compare the results for $N=50$ and $N=100$ at $T=1$-and become more pronounced on cooling toward $T_{\text {crys }}^{\text {hom }}$. However, the features of $C_{a b}^{2}$ discussed in the previous section, in particular the good agreement between the GFRC prediction and the simulation data, remain the same so long as $T>T_{\text {crys }}^{\text {hom }}$.

This state of affairs changes when the melt crystallizes. Previous work [14-16] revealed that CG-PVA chains shorter than $N \approx 50$ fully extend on crystallization, whereas longer chains fold back on themselves, forming lamellarlike crystalline structures that coexist with amorphous regions (cf. Fig. 2 ). For $N=10$ we thus expect and find-see inset of Fig. 8 - that $C_{a b}^{2}$ exhibits rodlike behavior, that is, $C_{a b}^{2}=1$ for all $|a-b|$. For $N=100$, on the other hand, the situation is different. $C_{a b}^{2}$ is negative for $0.38 \leqq|a-b| / N \lesssim 0.88$. A negative value of $C_{a b}^{2}$ implies that internal distances larger than expected from ideal chains occur so that $\left\langle r_{a b}^{4}\right\rangle$ exceeds the Gaussian result $5\left\langle r_{a b}^{2}\right\rangle^{2} / 3$. This finding is not unreasonable. Large internal distances can result from stretched chain sections which participate in the crystalline lamellae [36].

(b) Form factors. From Fig. 8 we may conclude that the form factor $w(q)$ for $N=100$ in the crystalline state cannot be described by the Koyama approximation because $C_{a b}^{2}$ is negative. The approximation could work, however, for

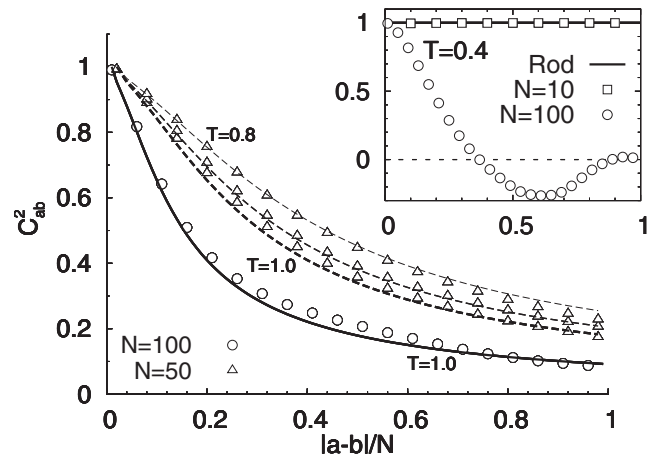

FIG. 8. Main figure: Non-Gaussian parameter $C_{a b}^{2}$ versus $|a-b| / N$ for $N=100$ at $T=1$ and for $N=50$ at $T=0.8,0.9$, and 1. All temperatures are above the onset temperature of homogeneous crystallization $T_{\text {crys }}^{\text {hom }}=0.78$. As in Fig. 7, the data shown represent gliding averages along the chain over all monomers $a$ which are separated by a contour length $|a-b|$ from monomer $b$ (i.e., $b=a+|a-b|$ ). Inset: $C_{a b}^{2}$ versus $|a-b| / N$ for $N=10$ and 100 in the semicrystalline phase at $T=0.4$. Perfect rodlike behavior, $C_{a b}^{2}=1$, is indicated by a horizontal solid line.

$N=50$ at $T>T_{\text {crys }}^{\text {hom }}$ where $C_{a b}^{2}>0$. This is indeed the case. Figure 9 compares simulation results for $w(q)$ in the $T$ interval $0.8 \leq T \leq 1$ with

$$
w^{K}(q)=\frac{1}{N} \sum_{a, b=1}^{N} w_{a b}^{K}(q),
$$

where we used Eq. (9) and $w_{a b}^{K}(q)$ is given by Eqs. (12) and (13). We find that $w^{K}(q)$ provides a good description of $w(q)$ for $T>T_{\text {crys }}^{\text {hom }}$.

In the crystalline state we can apply the Koyama approximation only to those cases for which $C_{a b}^{2}>0$. This condition is satisfied for short chains $(N=10)$ which form a crystal of rigid rods upon cooling. For rods, the Koyama approximation becomes exact, and the form factor reads

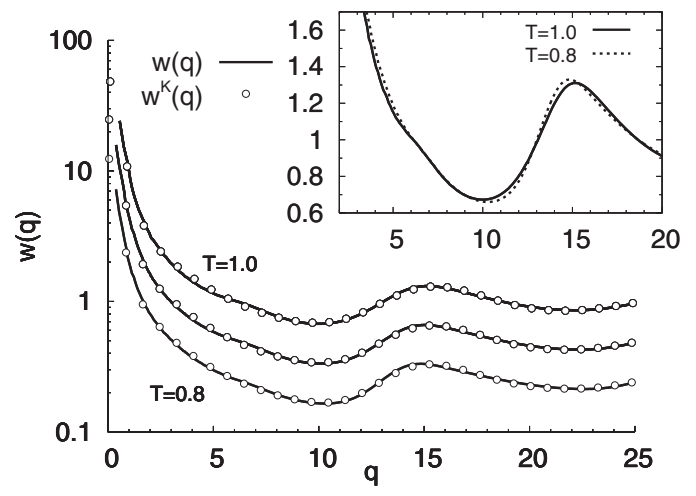

FIG. 9. Form factor $w(q)$ (lines) for $N=50$ chains at different temperatures, $T=0.8,0.9$, and 1 , above the onset temperature of homogeneous nucleation, $T_{\text {crys }}^{\text {hom }}=0.78$. Data for $T<1$ have been shifted downward by some factor for clarity $[w(q) / 2$ for $T=0.9$, $w(q) / 4$ for $T=0.8$ ]. The circles represent the Koyama approximation, Eq. (14). Inset: Comparison of the simulation results for $w(q)$ at $T=1.0$ and 0.8 . The slight shift of the peak at $q \approx 15$ toward lower $q$-values reflects the increase of chain stiffness upon cooling. 


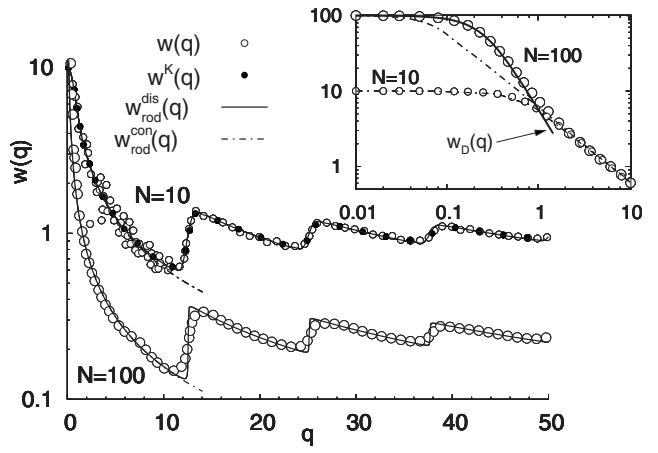

FIG. 10. Intrachain structure factor $w(q)$ (open circles) in the crystalline state $(T=0.4)$ for $N=10$ and 100 (data for $N=100$ are shifted). For $N=10$ we also show the Koyama approximation [closed circles, Eqs. (12)-(14)]. (For $N=100$ the approximation cannot be applied because $C_{a b}^{2}<0$ for some monomer pairs; cf. Fig. 8.) For both chain lengths the solid lines indicate the discrete version of the form factor of a rod, $w_{\text {rod }}^{\mathrm{dis}}(q)[\mathrm{Eq} .(15)]$ and the dashdotted lines the corresponding continuum limit, $w_{\text {rod }}^{\text {con }}(q)$ [Eq. (16) with $\left.R_{\mathrm{g}}=N b_{0} / \sqrt{12}\right]$. Inset: Small- $q$ behavior of the form factor. The simulation results are shown again by open circles. The dash-dotted lines indicate Eq. (16) with $R_{\mathrm{g}}=N b_{0} / \sqrt{12}$. For $N=100$, the solid line represents the Debye approximation $w_{D}(x)=2 N\left(e^{-x}-1+x\right) / x^{2}$ $[18,28]$, where $x=q^{2} R_{\mathrm{g}}^{2}$ and $R_{\mathrm{g}}$ denotes the radius of gyration obtained from the simulation.

$$
w^{K}(q) \equiv w_{\mathrm{rod}}^{\mathrm{dis}}(q)=\frac{1}{N} \sum_{a, b=1}^{N} \frac{\sin \left(b_{0}|a-b| q\right)}{b_{0}|a-b| q},
$$

if fluctuations of the bond length are neglected again [cf. Eq. (10)]. In the continuum limit Eq. (15) gives [18,37]

$$
w_{\text {rod }}^{\text {con }}(q)=\frac{N}{\sqrt{3} R_{\mathrm{g}} q} \int_{0}^{\sqrt{12} R_{\mathrm{g}} q} d t \frac{\sin t}{t}-N\left[\frac{\sin \left(\sqrt{3} R_{\mathrm{g}} q\right)}{\sqrt{3} R_{\mathrm{g}} q}\right]^{2} .
$$

Here, $R_{\mathrm{g}}=N b_{0} / \sqrt{12}$ is the radius of gyration of a rod.

We compare these predictions to the simulation results for $N=10$ and 100 at $T=0.4$ in Fig. 10. For both chain lengths the continuum expression Eq. (16) agrees with the simulated $w(q)$ for $q \gtrsim 1$. The discrete version of the form factor for a rod, Eq. (15), coincides with Eq. (16) for small $q$ and extends the description of the simulation data up to the largest $q$-values studied. The good agreement between the form factor of a rod and the simulation results for $N=10$ - not only for $q \gtrsim 1$, but also for smaller $q$-values (see inset of Fig. 10) - is expected, since short chains fully extend in the crystalline state. For $N=100$ the agreement for $q \gtrsim 1$ is, however, surprising. Long chains have a complex semicrystalline structure at low $T$ which should lead to deviations from perfect-rod behavior (and it does so for $q \lesssim 1$; see inset of Fig. 10). The finding " $w(q) \approx w_{\text {rod }}^{\text {dis }}(q)$ " for $q \gtrsim 1$ suggests that the crystalline structure of $N=100$ resembles a dense packing of rods. Apparently, the impact of the amorphous regions only manifests itself by smoothening the corners of the sawtoothlike structure of $w(q)$ compared to $w_{\text {rod }}^{\text {dis }}(q)$. For a wave vector smaller than $q=1$, the structural disorder present in the semicrystalline state becomes more apparent. Here, $w(q)$ is well-described by the Debye function $w_{D}(x)$ (see inset of Fig. 10), indicating that chain conformations on large length scales have Gaussian characteristics.

\section{Interchain structure factors}

As in the case of the intrachain structure we begin the discussion of intermolecular correlations by an analysis of the site-resolved quantities $h_{a b}(q)[\mathrm{Eq} .(7)]$ in the liquid state for $T>T_{\text {crys }}^{\text {hom }}$.

Figure 11 depicts $h_{a b}(q)$ at $T=1$ for $N=10$ and 100. If $q \gtrsim 17, h_{a b}(q)$ is close to the average pair-correlation function $h(q)$, the dependence on the monomer pair $(a, b)$ being very weak. For smaller $q$, however, we find a clear site-dependence. While pair correlations of inner sites $(1<a, b<N)$-with the exception of $h_{22}(q)$-remain close to each other and to $h(q)$, differences occur if chain ends $(a=1, N)$ are involved. Here, two observations can be made. First, at $q \approx q^{*}$, intermolecular correlations of chain ends are stronger than the average $h(q)$. Second, for $q \approx 4, h_{11}(q)$ exhibits a plateau, a feature not observed for $h(q)$.

A qualitatively similar behavior of $h_{a b}(q)$, albeit with weaker deviations of $h_{11}(q)$ from $h(q)$ for $q \gtrsim q^{*}$, was also found in previous work on a glass-forming bead-spring model [24]. For this model, the site-dependence of $h_{a b}(q)$ could be interpreted in terms of polymer reference interaction site model (PRISM) theory [27,38]. This prompts us to attempt the same analysis here.

\section{PRISM theory}

PRISM theory is a liquid state approach to the equilibrium properties of polymeric systems [27,38]. Its key idea is to decompose a polymer chain into spherical interaction sites. For bead-spring-like models, such as CG-PVA, the sites may be identified with the monomers.

Starting from this decomposition PRISM theory proposes a relation between the intrachain $\left[w_{a b}(q)\right]$ and interchain $\left[h_{a b}(q)\right]$ structure factors by a generalized Ornstein-Zernike equation $[23,27,38]$

$$
h_{a b}(q)=\sum_{x, y=1}^{N} w_{a x}(q) c_{x y}(q)\left[w_{y b}(q)+\rho_{\mathrm{ch}} h_{y b}(q)\right] .
$$

Here, $c_{a b}(q)$ is the direct correlation function between the sites $a$ and $b$. Physically, the direct correlation function may be interpreted as the effective pair potential between two monomer densities located on different chains in the melt [27]. In the homogeneous melt this intermolecular potential should depend only weakly on the specific position of the monomers $a$ and $b$ along the chains, provided they are not close to or at the chain ends. For long polymers, however, chain end effects should not be important, suggesting that all sites of a homopolymer can be treated as equivalent [39]. This implies

$$
c_{a b}(q)=c(q) .
$$

We test this equivalent-site approximation for $c_{a b}(q)$ in Appendix A. Here, we rather focus on its consequences for 

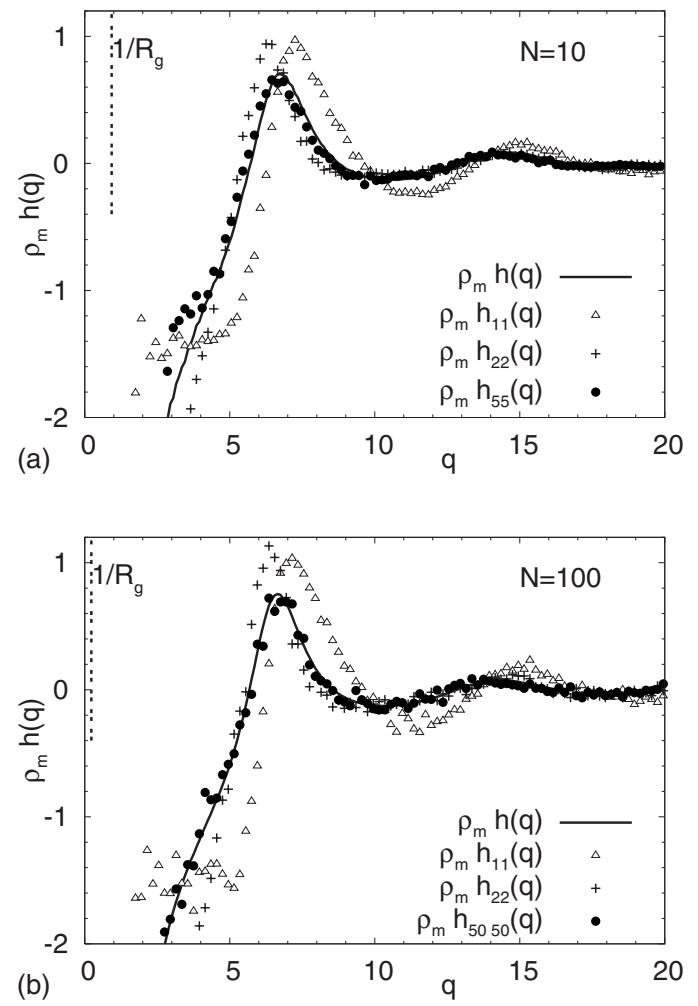

FIG. 11. Site-resolved $\left[h_{a b}(q)\right]$ and average $[h(q)]$ interchain structure factors at $T=1$ for two chain lengths, $N=10$ (upper panel) and $N=100$ (lower panel). Correlations involving both chain ends $(a=b=1)$ exhibit the strongest deviation from $h(q)$. For $a \geq 3$, the data for $h_{a a}(q)$ are close to or even coincide with $h(q)$. This is exemplified for the middle monomer $\left[h_{55}(q)\right.$ for $N=10$ and $h_{5050}(q)$ for $N=100]$. One observes that the agreement of $h_{a a}(q)$ and $h(q)$ improves with increasing $N$. The vertical dotted lines indicate the $q$-value corresponding to $R_{\mathrm{g}}\left(R_{\mathrm{g}} \simeq 1.08\right.$ for $N=10$ and $R_{\mathrm{g}} \simeq 4.46$ for $N=100)$.

$h_{a b}(q)$. Insertion of Eq. (18) into Eq. (17) and utilizing Eq. (A4) from Appendix A leads to [39]

$$
h_{a b}(q)=\frac{h(q)}{w(q)^{2}}\left[\sum_{x=1}^{N} w_{a x}(q)\right]\left[\sum_{y=1}^{N} w_{y b}(q)\right] .
$$

Thus PRISM theory predicts that the site-dependence of intermolecular correlations entirely results from the intrachain structure.

\section{Comparison with the simulation in the liquid phase}

Equation (19) is supposed to provide an approximate [39] representation of the site dependence of $h_{a b}(q)$ in the polymer liquid. Figure 12 tests this prediction. The figure compares Eq. (19) with the measured $h_{a b}(q)$ for $N=100$ at $T=1$. Contrary to the results of Ref. [24], where good agreement was obtained for all pairs $(a, b)$, we find here that $h_{a b}(q)$ is only well-described by Eq. (19) if $a, b \geq 3$. Pair correlations involving chain ends $(a=1)$ markedly deviate from the PRISM prediction (cf. first panel of Fig. 12). Deviations at the quantitative level are also visible for $h_{22}(q)$. Qualitatively, however, PRISM theory represents a substantial im-
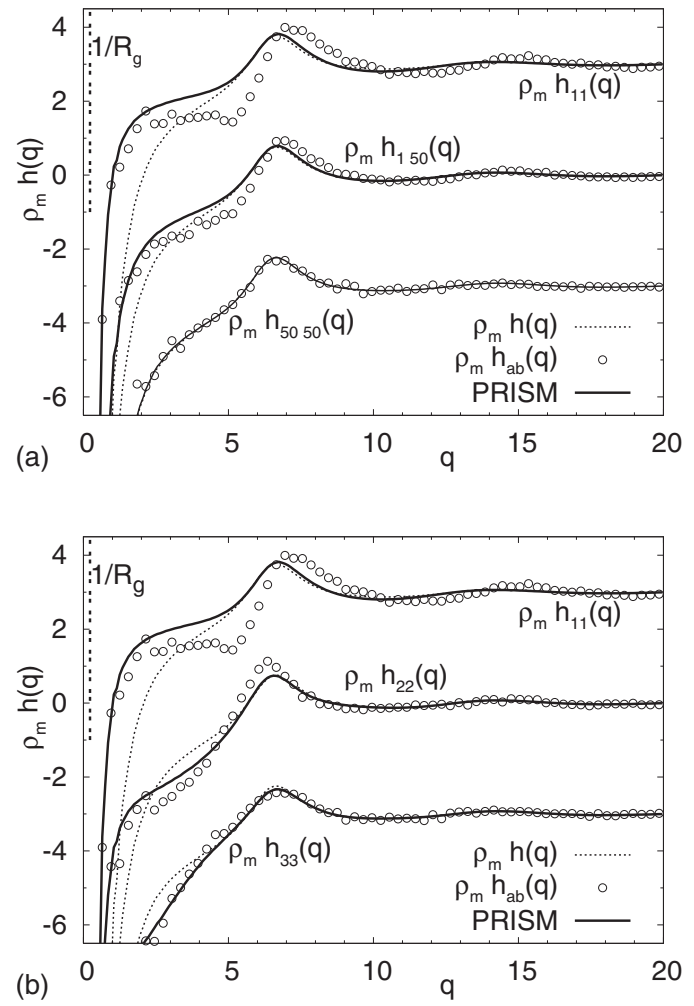

FIG. 12. Comparison of simulation results for the site-site interchain pair-correlation functions $h_{a b}(q)$ (open circles) with the PRISM prediction, Eq. (19) (solid lines). All data refer to $N=100$ and $T=1$. The input of Eq. (19), i.e., $w(q), h(q)$, and $w_{a b}(q)$, is also obtained from the simulation. The site-averaged correlation function $h(q)$ (dotted lines) is included for comparison. All curves except those for $h_{150}(q)$ and $h_{22}(q)$ are shifted vertically for clarity. The vertical dotted lines show the $q$-value corresponding to $R_{\mathrm{g}}\left(R_{\mathrm{g}}=4.46\right.$ for $\left.N=100\right)$.

provement as against the comparison of $h_{a b}(q)$ with $h(q)$ in Fig. 11. For instance, it provides a clear signature of the plateau for $h_{11}(q \approx 4)$ and is in general much closer to the simulated $h_{a b}(q)$ than is $h(q)$. These results are also found for other $T>T_{\text {crys }}^{\text {hom }}$ Thus we may obtain a semiquantitative description of the static structure of the CG-PVA polymer liquid by using the site-independent interchain direct correlation function $c(q)$ and the matrix of single-chain structure factors $w_{a b}(q)$.

\section{Temperature dependence of the interchain structure}

Below $T_{\text {crys }}^{\text {hom }}$ the melt is spatially heterogeneous; crystalline lamellae coexist with amorphous regions. It appears natural to assume that this heterogeneity modifies the intermolecular correlations between the monomers. Monomers participating in crystalline lamellae have different (local) environments-and thus different effective pair interactions - than those in the amorphous regions. So the equivalent-site approximation, Eq. (18), may be violated in the semicrystalline phase (see Appendix A for further discussion of this point), and the site dependence of $h_{a b}(q)$ cannot be understood in terms of Eq. (19). The following discussion 


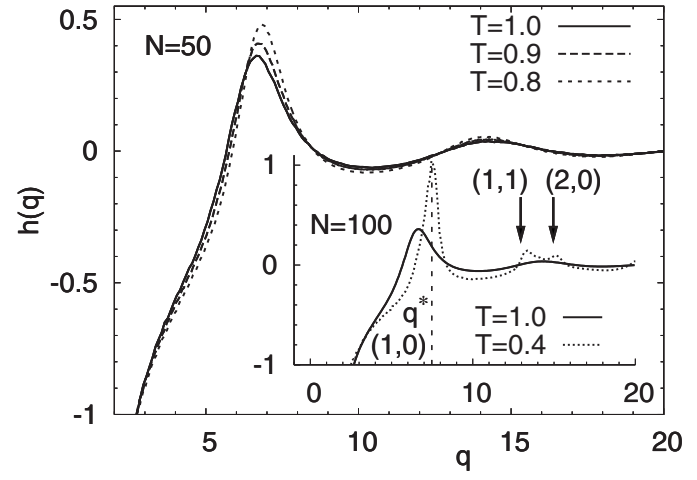

FIG. 13. Main figure: Site-averaged interchain structure factor $h(q)$ [Eq. (9)] for $N=50$ at different temperatures as indicated. Here, $h(q)$ is presented without the monomer density $\rho_{\mathrm{m}}$ as a prefactor. $q^{*}$ $(\approx 7.45)$ denotes the position of the first Bragg peak at $T=0.4$. Inset: Comparison of $h(q)$ in the liquid $(T=1)$ and crystalline $(T=0.4)$ states for $N=100$; results for $N=50$ are very similar. Bragg peaks of the (2D) hexagonal lattice are indicated by a vertical dashed line and arrows $\left[(1,0) \hat{=} q^{*} \approx 7.45,(1,1) \hat{=} \sqrt{3} q^{*}\right.$, and $\left.(2,0) \hat{=} 2 q^{*}\right]$.

thus focuses on the site-averaged intermolecular correlations $h(q)$.

Figure 13 depicts $h(q)$ for $N=50$ at various $T$ above $T_{\text {crys }}^{\text {hom }}$ and for $N=100$ also at $T=0.4$ below $T_{\text {crys }}^{\text {hom }}$. On cooling toward $T_{\text {crys }}^{\text {hom }}$ the intermolecular structure of the polymer liquid changes smoothly. The most prominent features are the shift of first peak at $q^{*}$ ("amorphous halo") to larger values and the increase of its amplitude $h\left(q^{*}\right)$. These changes are a consequence of the increase of density with decreasing $T$ and the attendant tighter intermolecular packing on the local scale of a monomer $\left(2 \pi / q^{*} \approx 1\right)$.

Below $T_{\text {crys }}^{\text {hom }}, h(q)$ shows the signature of Bragg peaks. For CG-PVA we expect to find a crystalline structure with hexagonal symmetry because elongated chain sections have a cylindrical shape. As we may assume the long axis of the cylinder to be much larger than the distance between cylinders, Bragg peaks should occur at the wave vectors of a two-dimensional hexagonal lattice, that is, at $q^{*}, \sqrt{3} q^{*}, 2 q^{*}$, etc. This expectation is borne out. We find peaks for these $q$-values, which are, however, substantially broadened because of the imperfect order of the semicrystalline state.

\section{Discussion: Structure factors in the liquid and semicrystalline states}

Important experimental quantities to explore structure and chain conformation of (crystallizable) polymer liquids are the (site-averaged) static structure factor $S(q)$ and form factor $w(q)$. Figure 14 recapitulates results from our simulations for these quantities, both in the liquid (upper panel) and crystalline states (lower panel).

In the liquid phase, $w(q)$ is almost independent of temperature, whereas $S(q)$ changes smoothly with decreasing $T$. Appealing back to Eq. (8) we see that this difference reflects modifications of the intermolecular packing. On cooling monomers (from different chains) have to pack more tightly to accommodate the increase of density. This compaction on

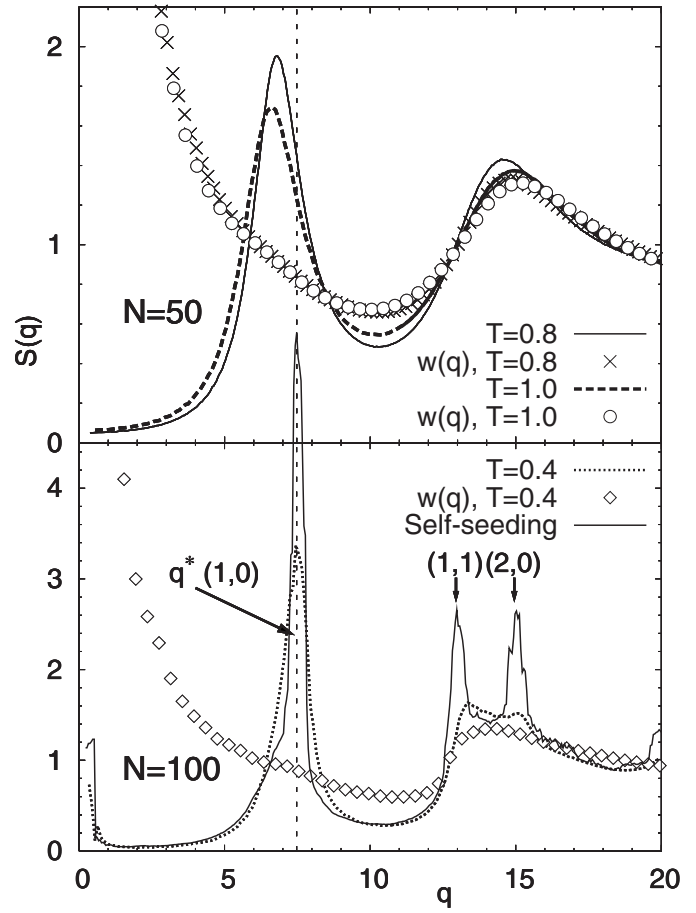

FIG. 14. Upper panel: Structure factor $S(q)$ (lines) and form factor $w(q)$ (symbols) versus $q$ for $N=50$ at two different $T$ above $T_{\text {crys }}^{\text {hom }}$, as indicated. Lower panel: $S(q)$ (dotted line) and $w(q)$ (diamonds) versus $q$ for $N=100$ in the semicrystalline phase at $T=0.4$. The solid line reproduces results from Ref. [15] obtained by the self-seeding process described in the text. $q^{*}(\approx 7.45)$ denotes the position of the first Bragg peak $(1,0)$. Higher order peaks of the (2D) hexagonal lattice are indicated by arrows $\left[(1,1) \hat{=} \sqrt{3} q^{*},(2,0)\right.$ $\left.\hat{=} 2 q^{*}\right]$.

a local scale entails a sharper spatial organization of the monomers in nearest-neighbor shells, the signature of which is the shift of the amorphous halo at $q^{*}$ and of its amplitude $S\left(q^{*}\right)$ to larger values. These features persist for all temperatures studied, even for $T$ very close to $T_{\text {crys }}^{\text {hom }}$ (e.g., $T=0.79$; not shown).

It is tempting to interpret this observation in terms of an empirical freezing criterion, the Hansen-Verlet criterion [40]. This criterion states that a liquid solidifies if $S\left(q^{*}\right)$ exceeds a critical value. For the freezing of simple liquids this value is $S\left(q^{*}\right) \approx 2.85[40-42]$. Contrary to that, we find $S\left(q^{*}\right) \approx 1.97$ at $T_{\text {crys }}^{\text {hom }} S\left(q^{*}\right)$ thus remains substantially below the established threshold for simple liquids [42], indicating that the polymer liquid crystallizes more easily than its monomeric counterpart.

This difference between simple and polymer liquids hints at the importance of the intrachain properties for the understanding of polymer crystallization. For simple liquids, crystallization may be interpreted as the result of a competition between liquid favoring, entropic forces (translational entropy) and solid favoring, packing forces. For polymer melts, this picture must be supplemented by intrachain contributions from the configurational entropy and internal energy of a chain. For instance, if the chains are fully flexible, they carry an enormous amount of entropy which must be lost upon crystallization. In general, intermolecular packing 
forces alone do not suffice to compensate for the entropy loss and to induce crystallization (except for specific cases [10]). If we removed the angular potential and kept only bond and LJ interactions, the CG-PVA model would not crystallize, but undergo a glass transition [43]. This demonstrates that it is the interplay of the (RIS-like) angular potential and the packing forces, which triggers crystallization in our model. Crystallization occurs if the packing constraints in the melt are sufficiently strong to assist the inherent tendency of the bonds to align colinearly. This straightens the chains and localizes the monomers on a crystalline lattice.

The features of the CG-PVA model imply that the expanded chain sections arrange parallel to each other on a hexagonal lattice in the semicrystalline phase. The structure factors for $N=100$ at $T=0.4$ in Fig. 14 exemplify this behavior. The $q$-dependence of $w(q)$ is close to the form factor of a rod, and $S(q)$ displays indications of Bragg peaks at the positions expected for a hexagonal lattice. The Bragg peaks are strongly broadened due to the imperfect crystalline structure. Better crystals may be obtained if we employ a "selfseeding process." In this process, the melt is first quenched to $T=0.7$ and tempered for a short time until nucleation occurs. Then, temperature is raised to $T=0.8$, and the system is further propagated [15]. During this isothermal relaxation only the largest crystalline nucleus survives; it serves as a heterogeneity for triggering crystallization. At $T=0.8$ the chains have sufficient mobility to better adapt to the crystalline pattern than during a continuous cooling process. Figure 14 shows that the Bragg peaks of the resulting $S(q)$ are much more pronounced. This clearly demonstrates the importance of chain mobility to attain better crystalline structures.

\section{SUMMARY AND CONCLUSIONS}

In this paper we discussed structural properties of a coarse-grained model for a crystallizable polymer melt at temperatures above and below the temperature $T_{\text {crys }}^{\text {hom }}$ of homogeneous crystallization. Our analysis employed various structure factors characterizing, on different length scales, intramolecular and intermolecular correlations between the monomers. We did not only determine average, but also sitespecific spatial correlations which depend on the monomer position along the chain backbone. Such detailed sitedependent information might be an important input in density-functional theories for polymer crystallization $[9,10]$. We hope that our work helps to further develop such approaches in the future. The main results of our work may be summarized as follows.

Above $T_{\text {crys }}^{\text {hom }}$ we find that the site-resolved intrachain structure factor $w_{a b}(q)$ is well-described by the Koyama distribution (Fig. 6). The input quantities of the Koyama distribution may be derived from a generalized freely rotating chain model (Fig. 7). So we can determine the site-dependence of $w_{a b}(q)$ by specifying only three conformational properties, the average bond length and the first and second moments of the cosine of the bond angle. Moreover, these results also imply that $w_{a b}(q)$ depends only on the distance $|a-b|$ between the monomers $(a, b)$ along the backbone (Fig. 5). This is in contrast to the intermolecular correlation functions $h_{a b}(q)$ which depend explicitly on the monomer pair $(a, b)$ (Fig. 11). The site-dependence of $h_{a b}(q)$ may be described semiquantitatively by PRISM theory (Fig. 12). PRISM theory assumes that the direct correlation function is independent of the monomer index. By calculating the site-site and site-averaged direct correlation functions we verify that this assumption is reasonable, unless chain ends are involved (Appendix A). This shows that even subtle intermolecular monomer-monomer correlations can be obtained, to a good approximation, from the knowledge of the site-averaged interchain direct correlation function and the site-dependent intrachain structure factors. Since our model thus allows one to describe intramolecular and intermolecular correlations in terms of established theoretical concepts, it might represent a convenient starting point for the development and test of liquid-state approaches to polymer crystallization.

Below $T_{\text {crys }}^{\text {hom }}$ short chains fully extend upon crystallization, whereas long chains form chain-folded lamellae separated by amorphous regions. The structural features of these polymer crystals lead to violations of the premises of the Koyama distribution-the non-Gaussian parameter $C_{a b}$ can become negative (Fig. 8) —or of PRISM theory-the equivalent-site approximation no longer applies (Fig. 17) — so that both theoretical approaches cannot be applied simultaneously.

Finally we also find that the polymer melt crystallizes more easily than a simple liquid because the Hansen-Verlet criterion, $S\left(q^{*}\right) \approx 2.85$, is not obeyed. This shows that the increase of packing constraints with decreasing $T$ is not the only driving force for crystallization in our model. It is crucial to take into account conformational properties-more precisely, backbone rigidity. Extended chain sections can pack more densely. The system thus gives up conformational entropy to satisfy packing constraints imposed by the increase of density on cooling below $T_{\text {crys }}^{\text {hom }}$. It is this coupling between the (RIS-like) backbone rigidity and density which drives the phase transition in our model. This interpretation suggests to study models with different rigidity in order to elucidate further these correlations between chain conformation and the tendency of the melt to crystallize. Such work as well as further analyses of the crystal phase are underway.

\section{ACKNOWLEDGMENTS}

We are indebted to M. Aichele and S.-H. Chong for many helpful discussions. Part of this work was made possible by a generous grant of computer time at the IDRIS (Orsay). Financial support by the MENESR, the DFG, the UFA (IRTG "Soft Condensed Matter"), the IUF, and the LEA MPIP-ICS is gratefully acknowledged.

\section{APPENDIX A: DIRECT CORRELATION FUNCTIONS}

In Sec. IV C 2 we introduced the site-dependent direct correlation function $c_{a b}(q)$ via the generalized OrnsteinZernike equation [Eq. (17)]

$$
h_{a b}(q)=\sum_{x, y=1}^{N} w_{a x}(q) c_{x y}(q)\left[w_{y b}(q)+\rho_{\mathrm{ch}} h_{y b}(q)\right],
$$

and assumed that 


$$
c_{a b}(q)=c(q) \quad(\text { equivalent }- \text { site approximation })
$$

The equivalent-site approximation - that is, the idea that the effective interaction potential between monomer densities of two different chains is independent of the specific monomer pair $(a, b)$-represents the key assumption of the PRISM theory $[27,38]$.

Here, we want to assess the range of validity of Eq. (A2) for the CG-PVA model in both the liquid and semicrystalline phases.

To test Eq. (A2) $c_{a b}(q)$ and $c(q)$ must be determined independently from the simulation. We obtain an expression for $c_{a b}(q)$ by inserting Eq. (3) into Eq. (A1). This yields

$$
\rho_{\mathrm{ch}} c_{a b}(q)=\left[w_{a b}^{-1}(q)-S_{a b}^{-1}(q)\right],
$$

where $X_{a b}^{-1}(q)$ denotes the $(a, b)$ element of the inverse matrix $X^{-1}(q)$ and $\rho_{\text {ch }}$ is the chain density. We can derive a similar expression for $c(q)$. Substituting Eq. (A2) into Eq. (A1) and making use of Eq. (9) leads to

$$
h(q)=w(q) c(q)\left[w(q)+\rho_{\mathrm{m}} h(q)\right]=w(q) c(q) S(q),
$$

from which we find $\left(\rho_{\mathrm{m}}=N \rho_{\mathrm{ch}}=\right.$ monomer density)

$$
\rho_{\mathrm{m}} c(q)=\frac{1}{w(q)}-\frac{1}{S(q)} .
$$

Equations (A3) and (A5) express $c_{a b}(q)$ and $c(q)$ in terms of the intrachain $\left[w_{a b}(q), w(q)\right]$ and interchain $\left[h_{a b}(q), h(q)\right]$ contributions of the static structure factors $S_{a b}(q)$ and $S(q)$ [Eqs. (4) and (8)]. Thus the direct correlation functions can be calculated from the simulation results discussed before.

(a) Direct correlation functions at $T=1$. Figure 15 tests the validity of the equivalent-site approximation for $N=10$ and 100 at $T=1$. We find that Eq. (A2) is well-satisfied, except for correlations involving the chain ends, $a=1$ or $a$ $=N$, and the monomers next to the ends, $a=2$ or $a=N-1$ (Fig. 16). The end-end correlations begin to deviate from $c(q)$ for $q \approx 15$. The deviations increase with decreasing $q$ and become particularly pronounced for $q \leqq q^{*}$ (cf. upper panel of Fig. 15). It is interesting to note that these deviations are not strongly influenced by chain length. They appear to be localized at the chain ends, regardless of how large $N$ is.

(b) Direct correlation functions at $T=0.4$. Short chains of length $N=10$ fully extend during crystallization (see Fig. 2). They form a polycrystalline structure consisting of domains in which the chains tend to arrange parallel to each other on a hexagonal lattice. This implies that monomers well inside the domains - e.g., the middle monomer of a chain-are surrounded by a crystalline environment, whereas those at the domain surfaces-e.g., the chain ends-are located in a more disordered environment. These differences should have a crucial impact on intermolecular correlations and so on the site dependence of the direct correlation functions. For instance, we may presume that the direct correlation function of the chain ends resembles the behavior found in the liquid phase, i.e., $c_{11}(q)$ is close to $c(q)$ for $q>5$, whereas $c_{55}(q)$ could substantially deviate from $c(q)$.
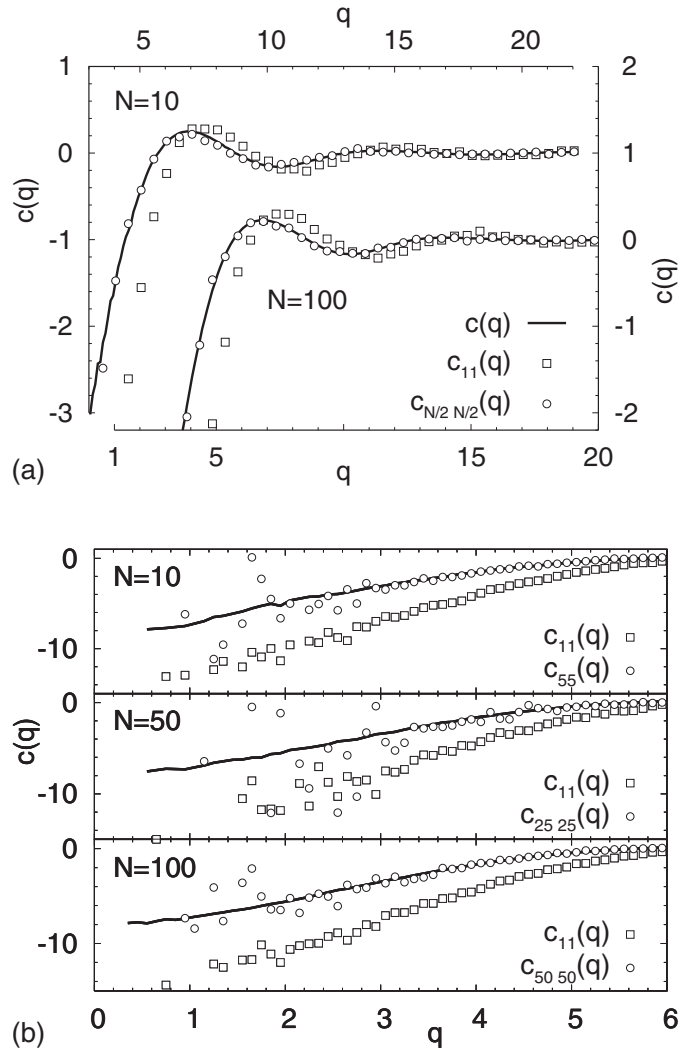

FIG. 15. Upper panel: Test of the equivalent-site approximation, $c_{a b}(q)=c(q)[$ Eq. $(\mathrm{A} 2)]$, at $T=1\left(>T_{\text {crys }}^{\text {hom }}=0.78\right) . c_{a b}(q)$ and $c(q)$ are determined from Eqs. (A3) and (A5), respectively. Results for two $N$ are shown, $N=10$ (left ordinate, bottom abcissa) and $N=100$ (right ordinate, top abcissa). For both $N$ the solid lines indicate $c(q)$ and the symbols represent $c_{a b}(q)$ for two monomer pairs $(a, b)$ : end-end correlations $(a=b=1)$ and center-center correlations $(a=b$ $=5$ for $N=10, a=b=50$ for $N=100$ ). Lower panel: Same comparison as in the upper panel, but with a focus on the small- $q$ regime; the direct correlation functions for $N=50$ are also included.

Figure 17 suggests that this conjecture is essentially correct. At $T=0.4$, we find indeed deviations of $c_{55}(q)$ from $c(q)$, which are not present at $T=1$ (Fig. 15) and which we thus attribute to the strongly heterogeneous structure of the



FIG. 16. Comparison of $c(q)$ (solid line) with $c_{a b}(q)$ (symbols) for monomer pairs involving the chain end and for $a=b=2$. It can be seen that the elements $c_{1 b}(q)$ differ from $c(q)$, particularly in the low- $q$ regime, even if $b=N / 2$. All data refer to $N=100$ and $T=1$. 


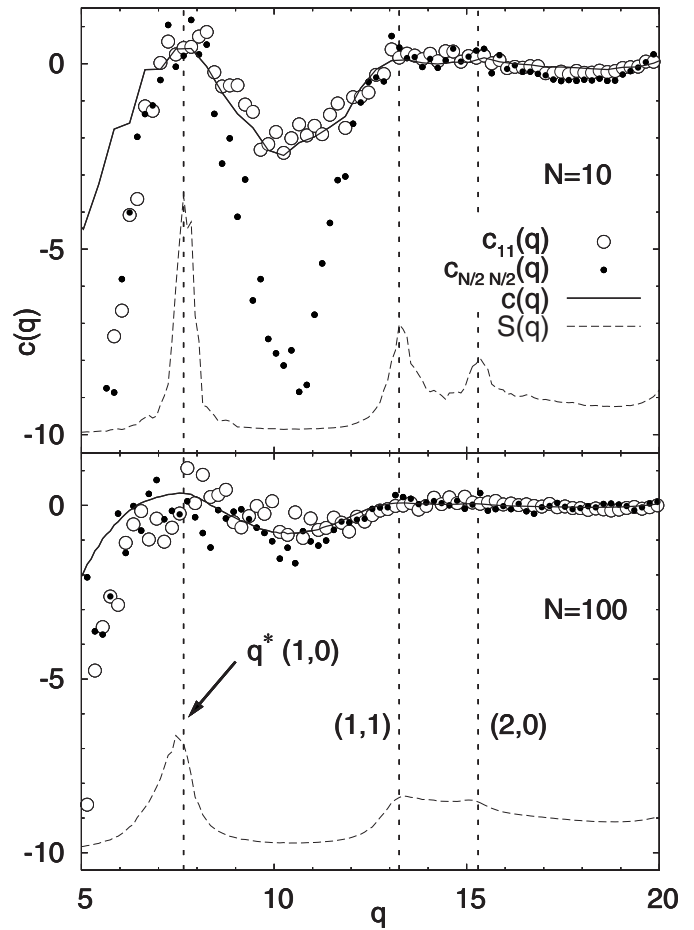

FIG. 17. Direct correlation functions in the semicrystalline phase $(T=0.4)$ for $N=10$ (upper panel) and $N=100$ (lower panel). For both $N$ the solid lines indicate $c(q)$ and the symbols represent $c_{a b}(q)$ for two monomer pairs $(a, b)$ : end-end correlations $(a=b$ $=1$ ) and center-center correlations $(a=b=5$ for $N=10, a=b=50$ for $N=100)$. In both panels the dashed lines show the static structure factor $S(q)$ which is shifted by -10 for clarity. $q^{*}(\approx 7.65)$ denotes the position of the first Bragg peak $(1,0)$ for $N=10$. Higher order peaks of the (2D) hexagonal lattice are indicated by arrows $[(1,1)$ $\hat{=} \sqrt{3} q^{*}$ and $\left.(2,0) \hat{=} 2 q^{*}\right]$.

short chain polymer crystal. For longer chains, the semicrystalline structure appears to be less heterogeneous. This is evidenced by a comparison of static structure factor for $N$ $=10$ and 100. Figure 17 shows that the Bragg peaks are strongly broadened and much less pronounced for $N=100$ than for $N=10$. We may therefore expect that the equivalence of all interaction sites is better respected for the longer
TABLE I. Values of the parameters $c$ and $p$ defined in Eq. (B1) measured from the simulation data for chains of length $N$ at temperature $T$.

\begin{tabular}{lccc}
\hline \hline$N$ & $T$ & $c$ & $p$ \\
\hline 100 & 1.0 & -0.68 & 0.19 \\
50 & 1.0 & -0.68 & 0.19 \\
50 & 0.9 & -0.72 & 0.27 \\
50 & 0.8 & -0.77 & 0.39 \\
\hline
\end{tabular}

chains. Despite the limited statistical accuracy of the data, Fig. 17 suggests that this is indeed the case.

\section{APPENDIX B: INTERNAL DISTANCES OF FREELY ROTATING CHAINS}

The Koyama distribution for the form factor is determined by the second and fourth moments of the distance distribution between two monomers $(a, b)$,

$$
\left\langle r_{a b}^{2}\right\rangle=\left\langle\left[\mathbf{r}^{a}-\mathbf{r}^{b}\right]^{2}\right\rangle \text { and }\left\langle r_{a b}^{4}\right\rangle=\left\langle\left[\mathbf{r}^{a}-\mathbf{r}^{b}\right]^{4}\right\rangle .
$$

For the model of a generalized freely rotating chain-that is, a single chain model with fixed bond length $b_{0}$ and a freely rotating bond angle $\theta$ that is not fixed, but fluctuates according to some distribution $P_{\text {ang }}(\theta)$-both moments can be expressed in terms of

$$
c=\langle\cos \theta\rangle, \quad p=\frac{1}{2}\left(3\left\langle\cos ^{2} \theta\right\rangle-1\right) .
$$

The second moment is a function of $c$ only $[19,44]$,

$$
\left\langle r_{a b}^{2}\right\rangle \equiv\left\langle r_{n}^{2}\right\rangle=n b_{0}^{2}\left(\frac{1-c}{1+c}+\frac{2 c}{n} \frac{1-(-c)^{n}}{(1+c)^{2}}\right) .
$$

Here, $n=|a-b|$ denotes the number of bonds between the monomers $(a, b)$. The result for the fourth moment $\left\langle r_{n}^{4}\right\rangle$ depends on $c$ and $p$. It reads [44]

$$
\left\langle r_{n}^{4}\right\rangle=\left\langle r_{n}^{2}\right\rangle^{2}+\frac{2}{3} b_{0}^{4} F_{n}
$$

with the following expression for the function $F_{n}$ :

$$
\begin{aligned}
F_{n}= & n^{2}\left(\frac{1-c}{1+c}\right)^{2}-n\left[1-\frac{2 c}{(1+c)^{3}}\left(6-5 c+3 c^{2}\right)-\left(\frac{1-c}{1+c}\right)^{2} \frac{4 p}{1-p}\right]-\left\{\frac{2 c}{(1+c)^{4}}\left(4-11 c+12 c^{2}\right)+\frac{4 p}{1-p}\left[1-\frac{8 c}{(1+c)^{3}}\right.\right. \\
& \left.\left.+\left(\frac{1-c}{1+c}\right)^{2} \frac{p}{1-p}\right]\right\}+(-c)^{n} \frac{8 c}{(1+c)^{3}}\left(n(1-3 c)+\frac{1-2 c+3 c^{2}}{1+c}-\frac{2 p}{(c+p)^{2}}\left[2 c^{2}+p c-p-n(1+c)(c+p)\right]\right)-\frac{6 c^{2 n+2}}{(1+c)^{4}} \\
& +p^{n}\left\{\frac{4}{1-p}\left[1-\frac{8 c}{(1+c)^{3}}-\left(\frac{1-c}{1+c}\right)^{2}\left(1-\frac{p}{1-p}\right)\right]+\frac{16 c^{2}}{(1+c)^{3}} \frac{c-c^{2}+2 p}{(c+p)^{2}}\right\} .
\end{aligned}
$$

Equations (B2) and (B4) allow us to compute $\left\langle r_{n}^{2}\right\rangle$ and the non-Gaussian parameter $C_{n}^{2}$ which enter the definition of the Koyama approximation for the form factor [Eqs. (12) and (13)]. The results of this calculation are compared to the simulation data in Figs. 7 and 8. Indicative values of $c$ and $p$ measured in the simulations can be found in Table I. 
[1] G. Strobl, The Physics of Polymers: Concepts for Understanding their Structures and Behavior (Springer, Berlin, 1997).

[2] Polymer Crystallization: Observations, Concepts and Interpretations, edited by J.-U. Sommer and G. Reiter, Lecture Notes in Physics Vol. 606 (Springer, Berlin, 2003).

[3] J. D. Hoffman and R. L. Miller, Polymer 38, 3151 (1997).

[4] M. Muthukumar, Adv. Chem. Phys. 128, 1 (2004).

[5] J. U. Sommer, Eur. Phys. J. E 19, 413 (2006).

[6] M. Al-Hussein and G. Strobl, Macromolecules 35, 1672 (2002).

[7] K. A. Armistead and G. Goldbeck-Wood, Adv. Polym. Sci. 100, 221 (1992).

[8] P. D. Olmsted, W. C. K. Poon, T. C. B. McLeish, N. J. Terrill, and A. J. Ryan, Phys. Rev. Lett. 81, 373 (1998).

[9] J. D. McCoy, K. G. Honnell, K. S. Schweizer, and J. G. Curro J. Chem. Phys. 95, 9348 (1991).

[10] N. Sushko, P. van der Schoot, and M. A. J. Michels, J. Chem. Phys. 115, 7744 (2001).

[11] D. W. Oxtoby, Annu. Rev. Mater. Res. 32, 39 (2002).

[12] J. Dzubiella and C. N. Likos, J. Phys.: Condens. Matter 15, L147 (2003).

[13] D. Reith, H. Meyer, and F. Müller-Plathe, Macromolecules 34, 2335 (2001).

[14] H. Meyer and F. Müller-Plathe, J. Chem. Phys. 115, 7807 (2001).

[15] H. Meyer and F. Müller-Plathe, Macromolecules 35, 1241 (2002).

[16] H. Meyer, Structure Formation and Chain-Folding in Supercooled Polymer Melts. Some Ideas from MD Simulations with a Coarse-Grained Model, Lecture Notes in Physics Vol. 606 (Springer, Berlin, 2003), pp. 177-195.

[17] T. Vettorel and H. Meyer, J. Chem. Theory Comput. 2, 616 (2006).

[18] M. Rubinstein and R. H. Colby, Polymer Physics (Oxford University Press, Oxford, 2003).

[19] P. J. Flory, Statistical Mechanics of Chain Molecules (Wiley, New York, 1969).

[20] B. Wunderlich, Macromolecular Physics, Vol. 1 (Academic, New York, 1973).

[21] M. P. Allen and D. J. Tildesley, Computer Simulation of Liquids (Clarendon Press, Oxford, 1994).

[22] M. Doi and S. F. Edwards, The Theory of Polymer Dynamics (Oxford University Press, Oxford, 1986).

[23] J. P. Hansen and I. R. McDonald, Theory of Simple Liquids (Academic Press, London, 1986).

[24] M. Aichele, S.-H. Chong, J. Baschnagel, and M. Fuchs, Phys. Rev. E 69, 061801 (2004).

[25] J. Baschnagel and F. Varnik, J. Phys.: Condens. Matter 17, R851 (2005).

[26] S. Krushev and W. Paul, Phys. Rev. E 67, 021806 (2003).

[27] K. S. Schweizer and J. G. Curro, Adv. Polym. Sci. 116, 319 (1994).

[28] P.-G. de Gennes, Scaling Concepts in Polymer Physics (Cornell University Press, Ithaca, 1996).

[29] A. Yethiraj, C. K. Hall, and K. G. Honnell, J. Chem. Phys. 93, 4453 (1990).

[30] A. Yethiraj and C. K. Hall, J. Chem. Phys. 96, 797 (1992).

[31] J. Melenkevitz, J. G. Curro, and K. S. Schweizer, J. Chem. Phys. 99, 5571 (1993).
[32] M. Schmidt and W. H. Stockmayer, Macromolecules 17, 509 (1984).

[33] M. L. Mansfield, Macromolecules 19, 854 (1986).

[34] The generalized freely rotating chain (GFRC) model represents a polymer as a sequence of $N-1$ bonds of fixed length, joined by a bond angle $\theta$ that fluctuates according to some distribution $P_{\text {ang }}(\theta)$ [19]. This model should provide a good approximation to the single chain properties of CG-PVA in the liquid phase due to the following reasons. (i) Bond-length fluctuations are strongly suppressed by the bond potential of CGPVA; the bond length is thus essentially fixed to its average value $b_{0}$. (ii) The distribution of torsional angles is (almost) uniform [43]. (iii) The distribution of bond angles, $P(\theta)$, obtained from the simulation almost coincides with the prediction $P_{\text {ang }}(\theta) \propto \sin \theta \exp \left[-U_{\text {ang }}(\theta) / k_{\mathrm{B}} T\right]$ which assumes that the distribution of $\theta$ is only determined by the bond-angle potential $U_{\text {ang }}(\theta)$. At $T=1$, this must be so by construction (cf. Sec. II). But we also find similar agreement between $P(\theta)$ and $P_{\text {ang }}(\theta)$ for lower $T$ provided the melt is in the liquid phase, $T>T_{\text {crys }}^{\text {hom }}$. See Ref. [17] for further discussion of this point.

[35] A caveat must be mentioned here. From the results presented in Fig. 7 one should not draw the conclusion that similar good agreement between simulation and the generalized freely rotating chain model can also be obtained for $N \gg 100$. For very long chains, there may be deviations from ideal Gaussian behavior, which are not related to microstructure effects, but to nonscreened excluded volume interactions between mesoscopic chain segments in the melt [46-48].

[36] In the semicrystalline phase, stretched chain sections can turn back upon themselves rather severely in order to fold into lamellae. Such conformations-extended chain portions interrupted by abrupt breaks - may lead to a violation of the inequality $C_{a b}^{2} \geq 0$, as noted by Mansfield in a different context [33].

[37] S. Stepanow, Eur. Phys. J. E 39, 499 (2004).

[38] D. R. Heine, G. S. Grest, and J. G. Curro, Adv. Polym. Sci. 173, 209 (2005)

[39] J. G. Curro and K. S. Schweizer, J. Chem. Phys. 87, 1842 (1987).

[40] J.-P. Hansen and L. Verlet, Phys. Rev. 184, 151 (1969).

[41] T. V. Ramakrishnan and M. Yussouff, Phys. Rev. B 19, 2775 (1979).

[42] F. Saija, S. Prestipino, and P. V. Giaquinta, J. Chem. Phys. 124, 244504 (2006).

[43] T. Vettorel, Ph.D. thesis, Université Louis Pasteur Strasbourg, 2005, available from http://eprints-scd-ulp.u-strasbg.fr:8080/ archive/00000334/.

[44] K. G. Honnell, J. G. Curro, and K. S. Schweizer, Macromolecules 23, 3496 (1990).

[45] A. Barbieri, E. Campani, S. Capaccioli, and D. Leporini, J. Chem. Phys. 120, 437 (2004).

[46] J. P. Wittmer, H. Meyer, J. Baschnagel, A. Johner, S. Obukhov, L. Mattioni, M. Müller, and A. N. Semenov, Phys. Rev. Lett. 93, 147801 (2004).

[47] S. P. Obukhov and A. N. Semenov, Phys. Rev. Lett. 95, 038305 (2005).

[48] P. Beckrich, A. Johner, A. N. Semenov, S. P. Obukhov, H. Benoît, and J. P. Wittmer, e-print cond-mat/0701261, Macromolecules (to be published). 